\title{
A simple clogging and backwashing efficiency model for filtration of arsenic- contaminated water
}

\begin{abstract}
Filtration is a very basic and primitive technique of water treatment. For many remote, underprivileged and poor communities, this is the only pre-treatment of drinking water prior to boiling. With the emergence of arsenic contaminations in many groundwater aquifers, the filtration became imperative for many communities around the world. However, after repetitive/continuous uses, clogging of the filter media is obvious, which eventually causes poor performance of the filtration process. Backwashing is a common technique being used for the recovery of the filtration capacity of clogged filter media. This study presents development of a simple clogging and backwashing efficiency model for a special filter media. "3rd generation IHE family filter" was developed by UNESCO-IHE Institute for Water Education and widely used for treating arsenic-contaminated water in many countries including Bangladesh. Several field tests were conducted in three different sites in Bangladesh having different qualities of influent water. Developed model coefficients were derived using the collected data on flow measurements through the device during successive clogging and backwashing periods up to four months. Developed model with the selected model coefficients can simulate field measurements on flow retardation and recovery with good accuracy. Eventually, selected model coefficients for three sites were correlated with the respective influent water quality. It was found that the coefficients are linearly correlated with the iron and ammonium contents of inflow water.
\end{abstract}

Keyword: Arsenic; Clogging; Backwashing; Filtration; Model 
